\title{
Staphylococcus aureus eye infections in two Indian hospitals: emergence of ST772 as a major clone
}

This article was published in the following Dove Press journal:

Clinical Ophthalmology

23 January 2012

Number of times this article has been viewed

\section{Savitha Nadig' \\ Nithya Velusamy ${ }^{2}$ \\ Prajna Lalitha ${ }^{2}$ \\ Sarita $\mathrm{Kar}^{3}$ \\ Savitri Sharma ${ }^{3}$ \\ Gayathri Arakere'}

'Society for Innovation and Development, Indian Institute of Science, Bengaluru, Karnataka, ${ }^{2}$ Aravind Eye Hospital, Madurai, Tamil Nadu, ${ }^{3}$ LV Prasad Eye Institute, Bhubaneswar, Odisha, India
Correspondence: Gayathri Arakere Society for Innovation and Development, Indian Institute of Science Campus, Bengaluru $5600 / 2$, India

Tel +9l 8023440139

$\mathrm{Fax}+918023466006$

Email gayathri.arakere@gmail.com
Purpose: The purpose of this study was to perform molecular characterization of Staphylococcus aureus isolates causing a variety of eye infections from two major eye care hospitals in India. Methods: Twenty-four isolates from Aravind Eye Hospital, Madurai, India, and nine isolates from LV Prasad Eye Institute, Bhubaneswar, India, representing severe to nonsevere eye infections like microbial keratitis to lacrimal sac abscess, were characterized. Staphylococcal cassette chromosome mec typing, multilocus sequence typing, accessory gene regulator typing, staphylococcal protein A typing, and pulsed field gel electrophoresis were used, along with determination of the presence of Panton-Valentine leucocidin toxin and endotoxin gene cluster among each sequence type.

Results: The majority of eye infections, both severe and nonsevere, were caused by sequence type (ST)772, positive for the Panton-Valentine leucocidin gene, and carrying methicillinresistant staphylococcal cassette chromosome mec type V cassette $(22 / 33,67 \%)$. Some of the other sequence types that caused severe eye infections were ST1 (9\%), $5(3 \%), 72(6 \%), 88(3 \%)$, $121(3 \%)$, and $672(3 \%)$. This is the first report of the presence of ST1 and 88 in India.

Conclusion: Although the number of isolates included in this study was small, most of the eye infections were caused by community-associated $S$. aureus where patients had no history of hospitalization or treatment in the past year. In the case of six severe infections, patients were admitted for surgeries and there is probability of hospital infection. In addition, only methicillin-resistant $S$. aureus isolates carrying staphylococcal cassette chromosome mec type V were detected. Epidemic methicillin-resistant Staphylococcus aureus 15 (ST22) is a major ST found in health care as well as community settings in non-eye infections in India, but only one methicillin-sensitive S. aureus isolate belonging to ST22 was detected. Predominantly ST772, along with a few other STs, caused the 33 eye infections studied.

Keywords: CA-MRSA, severe and nonsevere eye infections, ST772, PVL, agr type II

\section{Introduction}

Staphylococcus aureus is a common human pathogen responsible for a wide array of diseases from superficial skin infections to life-threatening pneumonia, septicemia, and endocarditis. ${ }^{1,2}$ This bacteria is also one of the most common ophthalmic pathogens recovered from conjunctivitis and other more serious ocular infections. ${ }^{3}$ The eye is a unique organ that is almost impermeable to all external agents. However, it can be infected from external sources or through intraocular invasion of micro-organisms carried by the blood stream. External bacterial infections of the eye are usually localized but may frequently spread to adjacent tissues, from the conjunctiva to the cornea, inner eye, orbit, or brain. Common eye infections caused by bacterial pathogens are blepharitis, conjunctivitis, internal and external hordeolum, microbial scleritis, 
canaliculitis, keratitis, dacryocystitis, preseptal cellulitis, orbital cellulitis, endophthalmitis, and panophthalmitis. It is estimated that more than 30,000 cases of microbial keratitis occur annually worldwide. Nonsurgical trauma and contactlens use are the leading predisposing risk factors for microbial keratitis. Timely institution of appropriate therapy must be initiated to control the infection and thereby minimize ocular morbidity and prevent blindness.

While methicillin-resistant S. aureus (MRSA) has been reported by the media as responsible for more deaths than acquired immune deficiency syndrome in the USA, very little is known about the prevalence and epidemiology of eye infections due to methicillin-sensitive $S$. aureus (MSSA) or MRSA. Methicillin resistance is conferred on the organism by the presence of a unique mobile genetic element called the staphylococcal cassette chromosome carrying the mecA gene ( $\mathrm{SCCmec}$ ). The SCCmec elements are divided into different types based on nucleotide differences in two essential components, (1) cassette chromosome recombinase ( $c c r$ ) represented by $c c r$ genes and mec gene complexes, and (2) also by variability in the joining regions. ${ }^{4,5}$ Until recently, only eight major types of SCCmec elements were reported, but three more types, of bovine and human origin, have now been added, increasing the total to eleven SCCmec types. ${ }^{6,7}$ Until a few years ago, hospital-associated MRSA (HA-MRSA) isolates contained mainly type I, II, and III SCCmec cassettes, while community-associated MRSA (CA-MRSA) contained type IV and V cassettes. But it is no longer possible to categorize $S$. aureus isolates as community or hospital associated based on their SCCmec types, as many hospitals around the world are seeing a rise in type IV and V SCCmec isolates. ${ }^{8}$

A recent Indian study reports that about $25 \%$ of ocular infections are caused by S. aureus in South India. ${ }^{9}$ However, there are no studies on the molecular characterization of types and clones of $S$. aureus causing ocular infections in India. In 2006, USA300 isolates causing severe ophthalmic manifestations were reported. ${ }^{10}$ More recently, Hesje et al have reported on the presence of both HA- and CA-MRSA isolates from ocular infections collected from 14 different states in the USA. ${ }^{11}$

This is the first report from India on the types and diversity of genotypes present among Indian ocular $S$. aureus isolates collected from two hospitals, one in the south and the other in the east.

\section{Methods}

\section{Bacterial strains}

$S$. aureus strains were obtained from different eye infections from two eye care hospitals: 24 specimens from
Aravind Eye Hospital (AEH), Madurai, South India, which serves as a primary and tertiary center, and nine specimens from LV Prasad Eye Institute (LVP), Bhubaneswar, Eastern India, serving mainly as a tertiary center.

\section{Patients}

The majority of the MRSA isolated from AEH were from patients with external adnexal diseases of the lacrimal sac abscess, lids, and orbit. There was no history of prior hospitalization for any of these patients. The samples were collected at the first visit before hospitalization. Three patients had more severe infections of postcataract endophthalmitis and microbial keratitis at AEH. Patients with postcataract endophthalmitis and buckle infection required hospitalization and vitrectomy. Corneal scrapings using platinum spatula were collected from all patients with microbial keratitis except one (exposure keratopathy) from whom a conjuctival swab was collected. For preseptal cellulitis samples, pus accumulated in the conjunctiva was collected. In the case of the patient with postcataract wound infection, pus was collected from the infected insertion site.

\section{Phenotypic characterization}

The isolates obtained from hospitals were selected on chromogenic agar medium (chromAgar, bioMerieux, France) and identified as $S$. aureus after Gram staining, detection of catalase, coagulase, and DNAse as described elsewhere. ${ }^{12}$

\section{Antibiotic susceptibility testing}

Susceptibility testing was performed by Kirby-Bauer disc diffusion according to the guidelines recommended by the Clinical Laboratory and Standards Institute ${ }^{13}$ on Mueller-Hinton agar plates (HIMEDIA, Mumbai, India) at $37^{\circ} \mathrm{C}$ using antibiotic discs. Minimum inhibitory concentration for oxacillin and cefoxitin was determined by the broth dilution method in Mueller-Hinton broth (HIMEDIA) after 24 hours of incubation at $37^{\circ} \mathrm{C}$ in microtiter plates. ${ }^{14}$

\section{Chromosomal DNA isolation}

Cells from an overnight culture in brain-heart infusion broth collected by centrifugation were suspended in TE buffer (10 mM Tris- $\mathrm{HCl}, \mathrm{pH} 8.0,1 \mathrm{mM}$ ethylenediaminetetraacetic acid, pH 8.0) containing 1500 u of achromopeptidase (Sigma Aldrich, Bommasandra, Bengaluru, India). The cell suspension was incubated at $37^{\circ} \mathrm{C}$ for 30 minutes, and an equal volume of phenol:chloroform (1:1) mixture was then added and the suspension was mixed vigorously in a vortex mixer. The samples were centrifuged and the aqueous phase was transferred to a fresh tube. The DNA was precipitated by addition of $30 \mu \mathrm{L}$ of 
$3 \mathrm{M}$ sodium acetate and three volumes of cold $99 \%$ ethanol. The DNA pellet was washed twice with cold $99 \%$ ethanol, air dried, and suspended in $500 \mu \mathrm{L}$ of TE buffer.

\section{Molecular characterization}

SCCmec typing was carried out by determination of the type of mec and $c c r$ complexes present in each MRSA isolate. They were determined by multiplex polymerase chain reaction (PCR) using published procedures and primers. ${ }^{15-17}$ Identification of SCCmec types IV and V was done by an updated multiplex PCR strategy for SCCmec typing by Milheirico et al. ${ }^{17}$

\section{Spa typing}

Spa typing was performed according to the procedure of Shopsin et al. ${ }^{18}$ Consensus sequences were matched from both forward and reverse sequences and the repeat units were identified using the Ridom database. ${ }^{19}$

\section{Multilocus sequence typing (MLST)}

MLST was done as described previously. ${ }^{20}$ Internal fragments of the seven housekeeping genes for carbamate kinase $(\operatorname{arc} C)$, shikimate dehydrogenase (aroE), glycerol kinase $(g l p F)$, guanylate kinase $(g m K)$, phosphate acetyltransferase (pta), triosephosphate isomerase (tpi), and acetyl coenzyme A acetyltransferase (yqiL) were amplified by PCR with the specified primers, and the PCR products were purified (QiaQuick PCR purification kit; QIAGEN GmbH, Hilden, Germany) and commercially sequenced (Macrogen, Inc, Seoul, South Korea). Consensus sequences were assembled from both the orientations. The allelic profile was matched using the Multi Locus Sequence Typing database. ${ }^{21}$

Detection of the $P V L$ gene and accessory gene regulator (agr) typing was carried out by PCR and multiplex PCR respectively. ${ }^{22,23}$ Enterotoxin gene cluster $(e g c)$ was detected by several multiplex PCRs. ${ }^{24}$

\section{Pulsed field gel electrophoresis (PFGE)}

PFGE was performed as described previously after restriction digestion using SmaI enzyme and a CHEF-DRIII (BioRad Laboratories [India] Private Limited, Bengaluru, India) device. $^{25}$

\section{Results}

\section{Clinical characterization}

Thirty-three $S$. aureus isolates from two eye care hospitals were isolated from 22 males and eleven females. Mean age was around 39 and 32 years for male and female patients, respectively. From LVP, there were eight severe cases (microbial keratitis) and one nonsevere (severe conjunctivitis); AEH had seven severe (three endophthalmitis, three microbial keratitis, and one buckle infection) and 17 nonsevere lid and adnexal infections. Table 1 illustrates the list of severe eye infections, predisposing factors, and comorbidities associated with patients. Among the seven severe infections from $\mathrm{AEH}$, four of them were admitted to the hospital - three for cataract surgery and one for retinal detachment. The endophthalmitis and buckle infection developed anywhere from two to four weeks after the surgery. The three other microbial keratitis patients from AEH had trauma to the eye from a foreign body (plant/soil), but had not been admitted to the hospital previously. All the other 17 patients with nonsevere infections from AEH had not been admitted to the hospital in the past year, nor had any surgery or treatment. Out of eight patients from LVP with severe infections, two patients (LVP1 and LVP8) had surgical repairs for corneal tear and acid injury. One patient with severe conjunctivitis (nonsevere infection) was suffering from retinoblastoma. The majority of the patients with lid and adnexal infections and microbial keratitis had infection and symptom resolution with appropriate antibiotics, while patients with postcataract endophthalmitis had poor prognoses.

\section{Molecular characterization}

Molecular characterization data from the $S$. aureus eye infections is presented in Table 2. Of 33 isolates, 22 (67\%) belonged to ST772, which is grouped under clonal complex (CC) 1, and 21 were MRSA. The remaining STs were 1, $5,22,30,72,88,121$, and 672 out of which ST72, 88, and 5 were MRSA and the rest MSSA. All the MRSA isolates (25/33) belonged to SCCmec type V. ST772 was associated with majority of the eye infections in the hospital from South India while the northeastern hospital had more diversity of CCs resulting in severe eye infections.

\section{ST772/CCI}

Among the $S$. aureus isolates causing ocular infections collected from two hospitals and genotyped in this study, 67\% of isolates (22/33) belonged to ST772 and SCCmec type V. The infections ranged from severe microbial keratitis to endophthalmitis to nonsevere lacrimal sac abscess, external hordeolum, preseptal cellulitis, and so forth. Representative eye infections are presented in Figure 1: (A) cellulitis, (B) endophthalmitis, (C) lacrimal sac abscess, (D) microbial keratitis, all caused by ST772 in four different subjects.

Except for one ST772 MSSA isolate causing microbial keratitis, all the rest were MRSA-carrying SCCmec type V. 


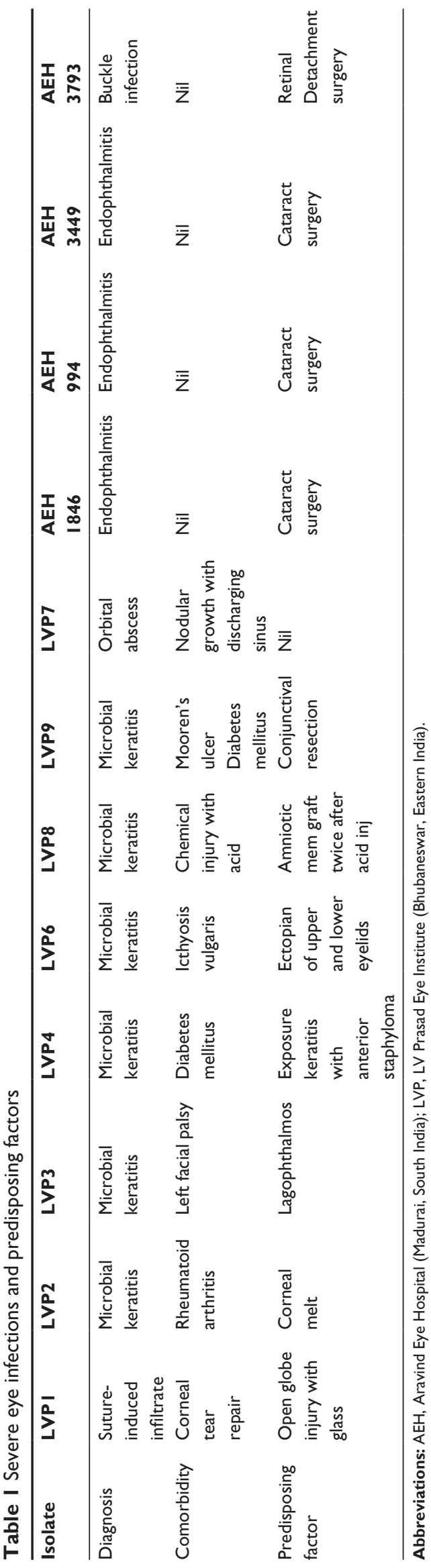

All isolates were Panton-Valentine leucocidin $(P V L)$ and $e g c$ positive, and belonged to accessory gene regulator $(a g r)$ type II. All ST772 isolates had spa type t657 except for one MRSA sample, which had spa type $\mathrm{t} 345$.

The antibiotic resistance pattern of all STs is presented in Table 3. Antibiotic sensitivity was checked for erythromycin, tetracycline, gentamicin, oxacillin, cefoxitin, chloramphenicol, cefazolin, gatifloxacin, ofloxacin, moxifloxacin, and ciprofloxacin. Out of 21 MRSA isolates, all were resistant to gentamicin, while resistance to erythromycin, oxacillin, cefoxitin, and cefazolin varied. The majority of ST772 MRSA isolates were also resistant to all the fluoroquinolone antibiotics tested. Except for seven isolates that caused severe infections like microbial keratitis and endophthalmitis, all other ST772 MRSAs were responsible for causing nonsevere soft tissue infections like lacrimal sac and upper lid abscesses.

\section{STI/CCI}

Three MSSA isolates from LVP causing severe infections of microbial keratitis and suture-induced infection from corneal tear repair belonged to ST1. This is the first report of ST1 S. aureus in India belonging to agr type III. All three isolates were $P V L$ and $e g c$ negative. Two isolates had the same spa type t127 and one had a new spa type t8078, which we have reported to the Ridom SpaServer database. ${ }^{19}$ Although ST772 and ST1 belong to CC1, they belong to two different $a g r$ types, II and III, respectively. All ST1 isolates were sensitive to gentamicin and tetracycline while resistance to erythmycin, oxacillin, and cefoxitin varied. Two ST1 isolates causing microbial keratitis were sensitive to chloramphenicol, cefazolin, and all the fluoroquinolones tested, while one ST1 isolate was resistant to ciprofloxacin alone.

\section{ST72/CC8}

There were two isolates of ST72 (double locus variant of ST8), one each of MSSA (LVP) and MRSA (AEH), causing microbial keratitis and buckle infection. In our previous study from the community and hospitals, we only isolated one MSSA isolate of ST72 while an MRSA was also detected among the eye isolates (unpublished data). Both isolates were $P V L$ negative and $e g c$ positive belonging to agr type I. MSSA was sensitive to all antibiotics while the resistance pattern of MRSA isolate was as shown in Table 3.

\section{STs $5,22,30,88, I 21$, and 672}

One isolate of each ST type was found among severe and nonsevere infections of the eye. ST 5, 88, and 672 were from LVP, causing microbial-keratitis, suture-induced, and socket 
Table 2 Molecular characterization of Staphylococcus aureus isolates from eye infections

\begin{tabular}{|c|c|c|c|c|c|c|c|c|c|}
\hline Infection & Sample & $\mathbf{N}(\%)$ & $\begin{array}{l}\text { Spa } \\
\text { type }\end{array}$ & ST/CC & $\begin{array}{l}\text { MRSA/MSSA } \\
\text { N/N }\end{array}$ & $\begin{array}{l}\text { SCCmec } \\
\text { type }\end{array}$ & Agr type & PVL & egc \\
\hline \multirow[t]{9}{*}{ Microbial keratitis } & Corneal scraping LVP & $9(27)$ & t8078* & $1 / 1$ & $0 / 1$ & & III & $-v e$ & $-v e$ \\
\hline & Corneal scraping LVP & & $\mathrm{t} 127$ & $\mathrm{I} / \mathrm{I}$ & $0 / 1$ & & III & $-v e$ & -ve \\
\hline & Corneal scraping LVP & & t657 & $772 / 1$ & $0 / 1$ & & II & $+\mathrm{ve}$ & $+\mathrm{ve}$ \\
\hline & Corneal scraping LVP & & $\mathrm{t} 345$ & $772 / 1$ & $1 / 0$ & V & II & + ve & +ve \\
\hline & Corneal scraping LVP & & $\mathrm{t} 442$ & $5 / 5$ & $\mathrm{I} / 0$ & v & II & $-\mathrm{ve}$ & $+\mathrm{ve}$ \\
\hline & Corneal scraping LVP & & $\mathrm{tl} 48$ & $72 / 8$ & $0 / 1$ & & I & -ve & $+\mathrm{ve}$ \\
\hline & Corneal scraping AEH & & $\mathrm{t} 657$ & $772 / 1$ & $1 / 0$ & V & II & +ve & +ve \\
\hline & Corneal scraping AEH & & $\mathrm{t} 657$ & $772 / 1$ & $1 / 0$ & V & II & +ve & +ve \\
\hline & Conjunctival swab AEH & & t657 & $772 / 1$ & $1 / 0$ & V & II & $+\mathrm{ve}$ & $+\mathrm{ve}$ \\
\hline Lacrimal sac abscess & Pus, conjunctival swab AEH & $5(15)$ & $\mathrm{t} 657$ & $772 / 1$ & $3 / 0$ & V & II & +ve & +ve \\
\hline $\begin{array}{l}\text { Orbital cellulitis secondary } \\
\text { to abscess }\end{array}$ & Pus, pus AEH & $2(6)$ & $\mathrm{t} 657$ & $772 / 1$ & $2 / 0$ & V & II & +ve & +ve \\
\hline Preseptal cellulitis & Pus, pus AEH & $2(6)$ & $\mathrm{t} 657$ & $772 / 1$ & $2 / 0$ & V & II & $+\mathrm{ve}$ & +ve \\
\hline Preseptal cellulitis & Conjunctival swab AEH & I (3) & t021 & $30 / 30$ & $0 / 1$ & & III & +ve & +ve \\
\hline Preseptal cellulitis & Pus AEH & I (3) & t005 & $22 / 22$ & $0 / 1$ & & 1 & +ve & +ve \\
\hline Upper-lid abscess & Pus AEH & $2(3)$ & $\mathrm{t} 657$ & $772 / 1$ & $2 / 0$ & V & II & $+\mathrm{ve}$ & +ve \\
\hline Conjunctivitis & Conjunctival swab AEH & I (3) & t657 & $772 / 1$ & $\mathrm{I} / 0$ & V & II & +ve & +ve \\
\hline Severe conjunctivitis & Conjunctival discharge LVP & I (3) & t1309 & 672 & $0 / 1$ & & 1 & $-v e$ & $+\mathrm{ve}$ \\
\hline \multicolumn{10}{|l|}{ Socket infection } \\
\hline External hordeolum & Pus AEH & I (3) & $\mathrm{t} 657$ & $772 / 1$ & $1 / 0$ & V & II & $+\mathrm{ve}$ & +ve \\
\hline Post-DCR wound infection & Pus AEH & I (3) & $\mathrm{t} 657$ & $772 / 1$ & $\mathrm{I} / 0$ & V & II & +ve & +ve \\
\hline Preseptal abscess & Pus AEH & I (3) & $\mathrm{t} 657$ & $772 / 1$ & $\mathrm{I} / 0$ & V & II & +ve & +ve \\
\hline Postcataract endophthalmitis & Vitreous biopsy AEH & $2(6)$ & $\mathrm{t} 657$ & $772 / 1$ & $1 / 0$ & $\vee$ & II & + ve & +ve \\
\hline Postcataract endophthalmitis & $\begin{array}{l}\text { Scraping from suture } \\
\text { infiltrate AEH }\end{array}$ & I (3) & $\mathrm{t} 2700$ & $|2| /|2|$ & $0 / 1$ & & IV & $-\mathrm{ve}$ & +ve \\
\hline Buckle infection & $\begin{array}{l}\text { Swab from exposed } \\
\text { buckle AEH }\end{array}$ & I (3) & $\mathrm{t} 8317^{*}$ & $72 / 8$ & $\mathrm{I} / 0$ & V & 1 & -ve & +ve \\
\hline Suture-induced infiltrate & Corneal scraping LVP & I (3) & $\mathrm{t} \mid 27$ & $\mathrm{I} / \mathrm{I}$ & $0 / 1$ & & III & -ve & $-\mathrm{ve}$ \\
\hline Orbital abscess & Purulent material LVP & I (3) & $\mathrm{t} 2526$ & $88 / 88$ & $\mathrm{I} / 0$ & V & III & $+\mathrm{ve}$ & $+\mathrm{ve}$ \\
\hline
\end{tabular}

Notes: -ve, negative; +ve, positive; *new spa types reported to the Ridom SpaServer database. ${ }^{19}$

Abbreviations: DCR, dacryocystorhinostomy; LVP, LV Prasad Eye Institute (Bhubaneswar, Eastern India); AEH, Aravind Eye Hospital (Madurai, South India); MRSA, methicillin-resistant S. aureus; MSSA, methicillin-sensitive S. aureus.
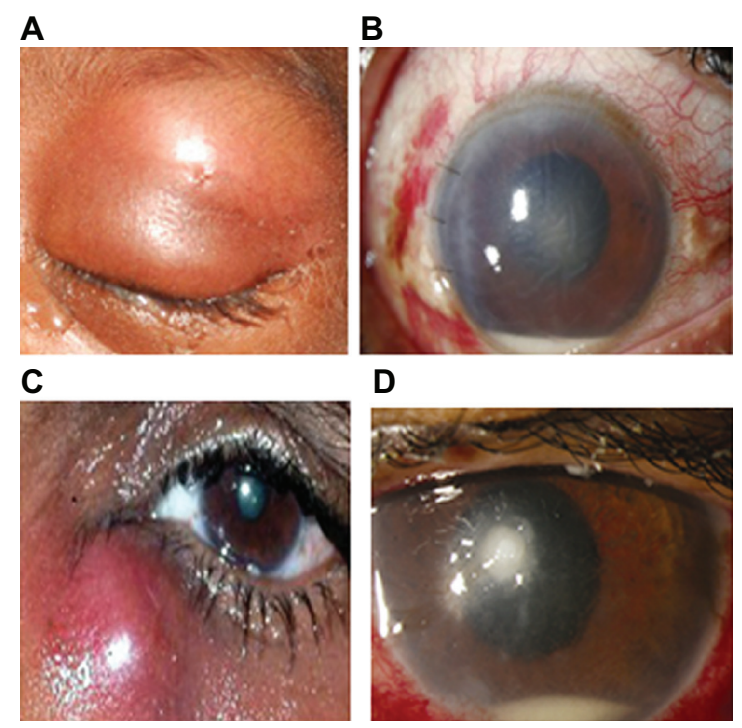

D

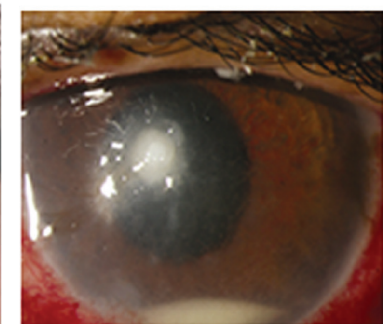

Figure I (A) Swollen erythematous eyelid in a case of orbital cellulitis; (B) slit-lamp picture of a case of endophthalmitis showing central corneal edema with hypopyon; (C) abscess of lacrimal sac secondary to acute dacryocystitis; (D) slit-lamp picture showing localized stromal infiltrate with hypopyon. infections. ST88 has been detected as MRSA for the first time in India. ST672 is an emerging clone found as MRSA and MSSA in non-eye infections also, from our previous study (unpublished data). While ST672 MSSA was resistant to chloramphenicol, the ST121 MSSA isolate causing endophthalmitis was sensitive to all antibiotics.

\section{PFGE patterns}

Figure 2A depicts the PFGE patterns of all the STs present among eye infections along with two ST772 isolates from skin and soft tissue infections. The PFGE patterns of ST772 isolates from eye infections and infections from other sites were nearly identical and closely related. Figure $2 \mathrm{~B}$ indicates the relatedness among the isolates in Figure 2A based on similarities derived from the unweighted pair group method with arithmatic mean (used to construct phylogenetic trees like the one in Figure 2B) and dice coefficients using Quantity $\mathrm{One}^{\circledR}$ 1-D Analysis Software (BioRad). 
Table 3 Antibiotic resistance patterns of Staphylococcus aureus isolates from eye infections from two Indian cities

\begin{tabular}{|c|c|c|c|c|c|c|c|c|c|c|c|c|c|}
\hline Eye infection & $\mathbf{C C} / \mathrm{ST}$ & $\begin{array}{l}\text { N MRSA/ } \\
\text { MSSA }\end{array}$ & Ery & Tet & Gen & Oxa & Cef & Chl & Cefaz & Gat & Ofl & Mox & Cipro \\
\hline Microbial & $\mathrm{I} / 772$ & I MSSA & II & IS & II & II & IS & IR & IS & IS & IS & IS & IS \\
\hline \multirow[t]{4}{*}{ keratitis } & $\mathrm{I} / 772$ & 4 MRSA & $2 S, I R, I I$ & $4 S$ & $4 \mathrm{R}$ & $2 \mathrm{R}, 2 \mathrm{I}$ & $4 R$ & $3 S, I R$ & $2 S, I R, I I$ & IS,3R & $4 \mathrm{R}$ & $4 \mathrm{R}$ & $4 \mathrm{R}$ \\
\hline & $1 / 1$ & $0 / 2$ & IS,IR & $2 S$ & $2 S$ & 21 & IS, II & $2 S$ & $2 S$ & $2 S$ & $2 S$ & $2 S$ & $2 S$ \\
\hline & $5 / 5$ & $1 / 0$ & IR & IS & IR & IR & IR & IS & IS & II & II & IR & IR \\
\hline & $8 / 72$ & $0 / 1$ & IS & IS & IS & IS & IS & IS & IS & II & IR & IR & IR \\
\hline $\begin{array}{l}\text { Lacrimal } \\
\text { sac abscess }\end{array}$ & I/772 & $5 / 0$ & IS,3I,IR & $5 S$ & $5 R$ & $3 \mathrm{I}, 2 \mathrm{R}$ & $5 R$ & $5 S$ & $5 S$ & $5 R$ & $5 R$ & $5 R$ & $5 R$ \\
\hline Orbital cellulitis & $\mathrm{I} / 772$ & $2 / 0$ & 21 & $2 S$ & $2 R$ & IS,II & $2 R$ & $2 S$ & $2 S$ & $2 R$ & $2 R$ & $2 R$ & $2 \mathrm{R}$ \\
\hline Preseptal & $\mathrm{I} / 772$ & $2 / 0$ & $I I, I R$ & $2 S$ & $2 R$ & $2 \mathrm{R}$ & $|R, I|$ & $2 S$ & $2 S$ & $2 \mathrm{R}$ & IS,IR & $2 \mathrm{R}$ & $2 \mathrm{R}$ \\
\hline \multirow[t]{2}{*}{ cellulitis } & $22 / 22$ & $0 / 1$ & IS & IS & IS & II & II & IS & IS & II & IS & IS & IS \\
\hline & $30 / 30$ & $0 / 1$ & II & IS & IS & IS & IS & IS & IS & IR & IR & IR & IR \\
\hline Upper lid abscess & $\mathrm{I} / 772$ & $2 / 0$ & 21 & $2 S$ & $2 \mathrm{R}$ & $I I, I R$ & $2 \mathrm{R}$ & $2 S$ & $2 S$ & $2 R$ & $2 R$ & $2 R$ & $2 R$ \\
\hline \multirow[t]{2}{*}{ Conjunctivitis } & I/772 & $1 / 0$ & II & IS & IR & $\mathrm{IR}$ & IR & IS & IS & IR & IR & IR & IR \\
\hline & $672 / 672$ & $0 / 1$ & IS & IS & IS & IS & IS & IR & IS & IS & IS & IS & IS \\
\hline External hordeolum & I/772 & $1 / 0$ & II & IS & IR & IR & IR & IS & IS & IR & IR & IR & IR \\
\hline Wound infection & $\mathrm{I} / 772$ & $1 / 0$ & II & IS & IR & IR & IR & IR & IS & IR & IR & IR & IR \\
\hline \multirow[t]{2}{*}{ Endophthalmitis } & $1 / 772$ & $2 / 0$ & 21 & $2 S$ & $2 R$ & $2 R$ & $2 R$ & $2 R$ & $2 S$ & $2 R$ & $2 R$ & $2 R$ & $2 R$ \\
\hline & $|2| /|2|$ & $0 / 1$ & IS & IS & IS & IS & IS & IS & IS & IS & IS & IS & IS \\
\hline Orbital abscess & $88 / 88$ & $1 / 0$ & IS & IS & IR & II & II & IS & IS & II & II & IR & IR \\
\hline Buckle infection & $8 / 72$ & $1 / 0$ & II & IS & IR & IR & IR & IS & IS & IR & IR & IR & IR \\
\hline $\begin{array}{l}\text { Suture-induced } \\
\text { infiltrate }\end{array}$ & $1 / 1$ & $0 / 1$ & IS & IS & IS & II & IS & IS & IS & IS & IS & IS & IR \\
\hline $\begin{array}{l}\text { Preseptal } \\
\text { abscess }\end{array}$ & $1 / 772$ & $\mathrm{I} / 0$ & IS & IS & IR & IR & IR & IS & IS & IR & IR & IR & IR \\
\hline
\end{tabular}

Abbreviations: MRSA, methicillin-resistant S. aureus; MSSA, methicillin-sensitive S. aureus; Ery, erythromycin; Tet, tetracycline; Gen, gentamicin; Oxa, oxacillin; Cef, cefoxitin; Chl, chloramphenicol; Cefaz, cefazolin; Gat, gatifloxacin; Ofl, ofloxacin; Mox, moxifloxacin; Cipro, ciprofloxacin; S, sensitive; R, resistant; I, intermediate.

\section{Discussion}

This is the first detailed study on the molecular characterization of $S$. aureus strains isolated from eye infections from two tertiary eye care hospitals in India. AEH, in South India, had predominantly ST772 (CC1) isolates. ST772 is the second major clone among CA-MRSA present in the Indian community and hospitals and belongs to $\mathrm{CC} 1{ }^{26}$ Work from our laboratory has shown that $19 \%$ of Indian isolates belonged to ST772 while $28 \%$ of isolates were EMRSA-15 (ST22) (unpublished data). Another Indian study has also shown EMRSA-15 and ST772 as major clones among patients in a tertiary care hospital in Mumbai. ${ }^{27}$

AEH patients with nonsevere infections (17) were not exposed to any health care settings in the previous year and had not undergone any treatment. Among the $S$. aureus isolated from these patients, 15 belonged to ST772 and one each to ST22 and ST30. These were of community origin and perhaps came from nasal carriage of the same isolates, but, as nasal swabs were not analyzed, it is not certain that the $S$. aureus causing the adnexal infections came from here. Perhaps the isolates were from a single outbreak. All ST772 isolates had somewhat similar antibiotic resistance patterns. Patients with severe infections at AEH listed in
Table 1 were admitted to the hospital for cataract surgery and retinal detachment. The endophthalmitis and buckle infection occurred 2-4 weeks after the cataract surgery and it is highly probable that the infections, caused by two isolates belonging to ST772, one to ST121, and one to ST72, were acquired in the hospital. All three microbial keratitis samples from AEH belonged to ST772; the patients with this infection had not been admitted to the hospital before and the infections were also of community origin. Nine isolates from LVP (Eastern India) were more diverse belonging to STs $1,88,5,672$ and 772, with only three being MRSA. All isolates except for one (socket infection) were from specimens with severe eye infections (Table 1). Among these, LVP1 patient ( ST1) had corneal tear repair surgery and LVP8 patient (ST72) had amniotic membrane graft twice due to acid injury, so it is possible that the infections were acquired in the hospital. With the other severe infections from LVP, there were comorbidities and it is hard to ascertain if the infections were hospital or community associated. There was more diversity in the sequence types among LVP patients. This is a tertiary care, specialty hospital where patients come from all over India for serious eye diseases, which perhaps explains the diversity found among the isolates. 
A

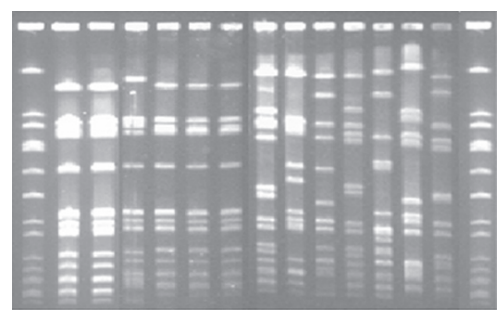

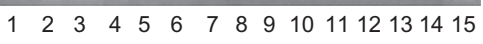

B
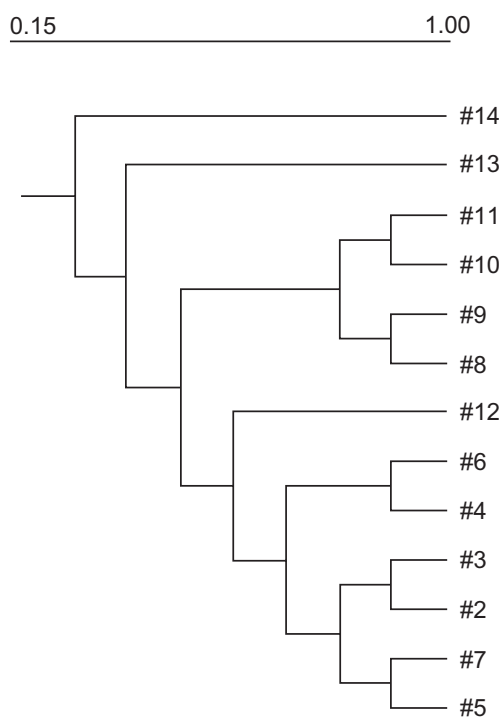

Figure 2 (A) Lanes I and 15, NCTC8325; Lanes 2, 3, 4, representative ST772 patterns from skin and soft tissue infections; Lanes 5, 6, 7, and 9, ST772 isolates from lacrimal sac abscess, orbital cellulitis, corneal ulcer and microbial keratitis respectively; Lanes 8 and II, STI isolates from microbial keratitis; Lanes I0, ST672 isolate from socket infection; Lane 12, ST88 isolate from orbital abscess; Lane 13, ST72 isolate from microbial keratitis; Lane 14, ST5 isolate from microbial keratitis. (B) Dendrogram showing the similarities derived from the unweighted pair group method with arithmatic mean and dice coefficients using the Quantity One ${ }^{\circledR}$ I-D Analysis Software.

The next important sequence type causing severe eye infections was ST1. Although the three ST1 isolates were methicillin sensitive, they amplified the downstream common sequence region of the SCCmec cassette (amplification product of $374 \mathrm{bp}$ in PCR), which is common for SCCmec types I, II, and IV. None of the other components of the SCCmec cassette, such as $c c r A B 2$ or $c c r C$, amplified. It is possible that the isolates contained a novel cassette without the mecA gene or that mecA and other parts of the cassette came off while the downstream common sequence region remained. ${ }^{28,29}$

ST1 is present in most parts of the world, while ST772 has been reported only in India, Malaysia, and Bangladesh. ${ }^{26,30,31}$ Although both ST772 and ST1 belong to CC1, their agr types are different and so are their virulence factors. The agr locus of $S$. aureus is a quorum-sensing gene cluster that upregulates production of secreted virulence factors and downregulates production of cell-associated virulence factors. ST772 isolates are positive for the presence of $P V L$ and $e g c$ genes, while ST1 isolates are negative. We have not done a detailed study of the virulence and immune-evasive factors present in the two, but they seem to have evolved differently. ST772 appears to have more fitness for infection of different tissues in our region. All MRSA isolates carried only SCCmec type $\mathrm{V}$ cassettes and close to $80 \%$ of the isolates tested were $P V L$ and $e g c$ positive, which is of serious concern. Although the role of PVL is not completely elucidated, epidemiological studies show that combination of $P V L$ and $e g c$, along with other entero-toxins, could increase the severity of diseases caused by $S$. aureus.

Among the other STs, ST5, 72, and 88 have manifested as MRSA, causing severe eye infections. These were resistant to all antibiotics except tetracycline, chloramphenicol, and cefazolin. All three STs have type V SCCmec cassettes while only ST88 is PVL positive. As eye injuries are very frequently treated with topical antibiotics, the chance of antibiotic-resistance development among microorganisms present is high. It is also possible that indiscriminate use of antibiotics in the community allows resistant strains to colonize eyes in the general population in the community. Recently, Ghebremedhin et al have reported a number of ST88 MRSA isolates causing conjunctivitis from Nigeria and have characterized it as carrying a type IV SCCmec cassette and as PVL positive. ${ }^{32}$ Horizontal transfers of different $\mathrm{SCCmec}$ cassettes could be occurring with isolates belonging to the same ST or CC.

A serious limitation in this study is the small number of isolates studied and also the fact that nasal swabs were not analyzed for the presence of $S$. aureus before treatment. There was twice the number of male patients with eye infections compared with the number of female patients. Again, due to the small sample size, it is not possible to come to a conclusion about this finding.

This effort was an attempt to study the eye infections from S. aureus with a view to comparing the sequence types causing eye and non-eye infections, their origins, and differences. We have been engaged in molecular characterization of S. aureus isolates causing skin and systemic infections and, from this study, it is clear that many of the sequence types are common among $S$. aureus infections except for ST1 and 88 , which we have not previously found.

From earlier studies of molecular characterization of S. aureus isolates in India, EMRSA-15, belonging to ST22, was one of the most prominent STs present in the Indian community as well as hospitals. ${ }^{26,27,33}$ Surprisingly, we 
found only one ST22 among AEH isolates causing preseptal cellulitis. The PFGE pattern of this ST22 isolate resembled an EMRSA-15 pattern from the nasal swab of a carrier from our collection. ${ }^{33}$ The sample size was too small to conclude that EMRSA-15 is limited in causing eye infections. However, it is clear that ST772 has an edge in causing eye infections in the community and perhaps in the hospital also. Among 33 isolates characterized, none belonged to sequence type ST239, which was the major HA-MRSA a few years ago. ST22 and 772 clones seem to have progressively replaced the ST239 clone, a clone associated with hospital-associated infections. ${ }^{25,27}$

More work is needed in the area of tissue specificity of certain STs of $S$. aureus in causing infections. This study suggests that ST772 is responsible for the majority of eye infections. However, several other STs causing serious eye infections are also reported in this paper.

\section{Acknowledgment}

GA is indebted to Department of Biotechnology (BT/IN/ New Indigo/16/GA/2010) Government of India, for financial support.

\section{Disclosure}

The authors report no conflicts of interest in this work.

\section{References}

1. Chambers HF, De Leo FR. Waves of resistance: Staphylococcus aureus in the antibiotic era. Nature Rev Microbiol. 2009;7:629-641.

2. Feng Y, Chen CJ, Su LH, Hu S, Yu J, Chiu C. Evolution and pathogenesis of Staphylococcus aureus: lessons learned from genotyping and comparative genomics. FEMS Microbiol Rev. 2008;32(1):23-37.

3. Behlau I, Gilmore MS. Microbial biofilms in ophthalmology and infectious disease. Arch Ophthamol. 2008;126(11):1572-1581.

4. Ito T, Katayama Y, Asada K, et al. Structural comparison of three types of staphylococcal cassette chromosome mec integrated in the chromosome in methicillin resistant Staphylococcus aureus. Antimicrob Agents Chemother. 2001;45(5):1323-1336.

5. Okuma K, Iwakawa K, Turnidge JD, et al. Dissemination of new methicillin-resistant Staphylococcus aureus clones in the community. J Clin Microbiol. 2002;40(11):4289-4294.

6. Li S, Skov RL, Han X, et al. Novel types of staphylococcal cassette chromosome mec elements identified in CC398 methicillin resistant Staphylococcus aureus strains. Antimicrob Agents Chemother. 2011;55: 3046-3050.

7. Shore AC, Deasy EC, Slickers P, et al. Detection of Staphylococcal cassette chromosome mec type XI carrying highly divergent mecA, mecI, mecR1, blaZ, and ccr genes in human clinical isolates of clonal complex 130 methicillin-resistant Staphylococcus aureus. Antimicrob Agents Chemother. 2011;55(8):3765-3773.

8. Popovich KJ, Weinstein RA, Hota B. Are community associated methicillinresistant Staphylococcus aureus (MRSA) strains replacing traditional nosocomial MRSA strains? Clin Infect Dis. 2008;46:787-794.

9. Ramesh S, Ramakrishnan R, Jayahar Bharathi M, Amuthan M, Viswanathan S. Prevalence of bacterial pathogens causing ocular infections in South India. Indian J Pathol Microbiol. 2010;53(2):281-286.
10. Rutar T, Chambers HF, Crawford JB, et al. Ophthalmic manifestations of infections caused by the USA 300 clone of community associated methicillin resistant Staphylococcus aureus. Ophthalmology. 2006;113: 1455-1462.

11. Hesje CK, Sanfilippo CM, Haas W, Morris TW. Molecular epidemiology of methicillin-resistant and methicillin-susceptible Staphylococcus aureus isolated from the eye. Curr Eye Res. 2011;36(2):94-102.

12. Baird D. Staphylococcus: cluster forming gram positive cocci. In: Collee JG, Fraser AG, Marmion BP, Simmons A, editors. Mackie and McCartney Practical Medical Microbiology. 14th ed. Vol 2. London: Churchill Livingstone; 1996:245-261.

13. Clinical and Laboratory Standards Institute. Performance Standards for Antimicrobial Susceptibility Testing, 19th Informational Supplement. M100-S19. Wayne, PA: Clinical and Laboratory Standards Institute; 2009.

14. Wikler MA, et al; National Committee for Clinical and Laboratory Standards Institute. Performance Standards for Antimicrobial Susceptibility Testing: Fifteenth Informational Supplement. Wayne, PA: Clinical and Laboratory Standards Institute; 2005.

15. Oliveira DC, de Lencastre H. Multiplex PCR strategy for rapid identification of structural types and variants of the mec element in methicillin-resistant Staphylococcus aureus. Antimicrob Agents Chemother. 2002;46(7):2155-2161.

16. Kondo Y, Ito T, Ma XX, et al. Combination of multiplex PCRs for staphylococcal cassette chromosome mec type assignment: rapid identification system for mec, ccr, and major differences in junkyard regions. Antimicrob Agents Chemother. 2007;51(1):264-274.

17. Milheirico C, Oliveira DC, de Lencastre, H. Update to the multiplex PCR strategy for the assignment of mec element types in Staphylococcus aureus. Antimicrob Agents Chemother. 2007;51(9):3374-3377.

18. Shopsin B, Gomez M, Montgomery SO, et al. Evaluation of protein A gene polymorphic region DNA sequencing for typing of Staphylococcus aureus strains. J Clin Microbiol. 1999;37(11):3556-3563.

19. Ridom Bioinformatics. Ridom SpaServer [database on the Internet]. Würzburg: Ridom GmbH; nd. Available from: http://SpaServer.ridom. de. Accessed December 7, 2011.

20. Enright MC, Day NP, Davies CE, Peacock SJ, Spratt BG. Multilocus sequence typing for characterization of methicillin resistant and methicillin susceptible clones of Staphylococcus aureus. J Clin Microbiol. 2000;38(3):1008-1015.

21. Multi Locus Sequence Typing: Staphyloccus aureus [database on the Internet]. Available at: http://saureus.mlst.net. London: Imperial College; nd. Accessed December 7, 2011.

22. Lina G, Piemont Y, Godail-Gamot F, et al. Involvement of PantonValentine leukocidin-producing Staphylococcus aureus in primary skin infections and pneumonia. Clin Inf Dis. 1999;29(5):1128-1132.

23. Gilot P, Lina G, Cochard T, Poutrel B. Analysis of the genetic variability of genes encoding the RNA III-activating components Agr and TRAP in a population of Staphylococcus aureus strains isolated from cows with mastitis. J Clin Microbiol. 2002;40(11):4060-4067.

24. Jarraud S, Cozon G, Vandenesch F, Bes M, Etienne J, Lina G. Involvement of enterotoxins $\mathrm{G}$ and I in staphylococcal toxic shock syndrome and staphylococcal scarlet fever. J Clin Microbiol. 1999; 37(8):2446-2449.

25. Arakere G, Nadig S, Swedberg G, Macaden R, Amarnath S, Raghunath D. Genotyping of methicillin resistant Staphylococcus aureus strains from two hospitals in Bangalore, South India. J Clin Microbiol. 2005;43(7):3198-3202.

26. Nadig S, Sowjanya SV, Seetharam S, Bharathi K, Raghunath D, Arakere G. Molecular characterization of Indian methicillin resistant Staphylococcus aureus. In: Raghunath D, Nagaraja V, Durga Rao C, editors. Proceedings of the 9th Sir Dorabji Tata Symposium on Antimicrobial Resistance - The Modern Epidemic Current Status and Research Issues. March 10-11, 2008; Bangalore: Macmillan Publishers India Ltd; 2008:167-184.

27. D'Souza N, Rodrigues C, Mehta A. Molecular characterization of methicillin-resistant Staphylococcus aureus with emergence of epidemic clones of sequence type ST 22 and ST 772 in Mumbai, India. J Clin Microbiol. 2010;48(5):1806-1811. 
28. Van der Zee A, Roorda L, Hendriks DH, Ossewaarde JM, Buitenwerf J. Detection of novel chromosome-SCCmec variants in Methicillin Resistant Staphylococcus aureus and their inclusion in PCR based screening. BMC Res Notes. 2011;4:150.

29. Mongkolrattanothai K, Boyle S, Murphy TV, Daum RS. Novel non-mecA containing staphylococcal chromosomal cassette composite island containing pbp4 and tagF genes in a commensal staphylococcal species: a possible reservoir for antibiotic resistance islands in Staphylococcus aureus. Antimicrob Agents Chemother. 2004;48(5):1823-1836.

30. Afroz S, Kobayashi N, Nagashima S, et al. Genetic characterization of Staphylococcus aureus isolates carrying Panton-Valentine Leukocidin genes in Bangladesh. Jpn J Infect Dis. 2008;61(5):393-396.
31. Ghaznavi-Rad E, Shamsudin MN, Sekawi Z, et al. Predominance and emergence of clones of hospital-acquired methicillin-resistant Staphylococcus aureus in Malaysia. J Clin Microbiol. 2010;48(3):867-872.

32. Ghebremedhin B, Olugbosi MO, Raji AM, et al. Emergence of a community-associated methicillin-resistant Staphylococcus aureus strain with a unique resistance profile in Southwest Nigeria. J Clin Microbiol. 2009;47(9):2975-2980.

33. Nadig S, Ramachandraraju S, Arakere G. Epidemic methicillin-resistant Staphylococcus aureus (EMRSA-15) variants detected in healthy and diseased individuals in India. $J$ Med Microbiol. 2010;59(Pt 7) $815-821$.
Clinical Ophthalmology

\section{Publish your work in this journal}

Clinical Ophthalmology is an international, peer-reviewed journal covering all subspecialties within ophthalmology. Key topics include: Optometry; Visual science; Pharmacology and drug therapy in eye diseases; Basic Sciences; Primary and Secondary eye care; Patien Safety and Quality of Care Improvements. This journal is indexed on

Submit your manuscript here: http://www.dovepress.com/clinical-ophthalmology-journal

\section{Dovepress}

PubMed Central and CAS, and is the official journal of The Society of Clinical Ophthalmology (SCO). The manuscript management system is completely online and includes a very quick and fair peer-review system, which is all easy to use. Visit http://www.dovepress.com/ testimonials.php to read real quotes from published authors. 